DOSAGE OF LIQUID MEDICINES: A SIMPLE PLAN FOR GREATER ACCURACY AND METRIC MEASURES.*

CARL S. N. HAILLBERG, PH.G.

Member Committee of Revision, U. S. Pharmacopeia ; Professor of Pharmacy in the School of Pharmacy, University of lllinols. CIIICAGO.

The strongest reason for the adoption of the metric weights and measures is the use of the present collection of arbitrary standards, conceived by chance and inherited by ages of medieval crudescence.

The paramount quality of that by which quantities are determined must be accuracy; that by which comparison is made must be as nearly invariable and inflexible as can possibly be devised, otherwise it is not a true standard. Scientific accuracy requires also that a fixed relation, only varied through variation in temperature, should prevail between weight and volume, and consequently that units of weights and liquid measures must be correlated, that is commensurate. No scheme for comparison which does not meet these fundamental requirements is worthy the name of a system, nor should be tolerated in this twentieth century.

\section{CUSTOMARY MEASURES.}

Subjecting the customary weights and liquid measures to this test they are found to be so absolutely at variance as to be a travesty on our boasted civilization and progress. Take the ounces of the three alleged systems for comparison, and they show the following equivalents in grains: avoirdupois, $43 \% 1 / 2$ grs.; apothecaries', or troy, 480 grs.; fluidounce, 456 grs. (water at $15 \mathrm{C}$.$) . This is due to the fact that one pint of water$ weighs 7,300 grs. (approx.) instead of one avoirdupois pound, or 7,000 grs. In other words, the old saying "A pint's a pound, the world around," is as far from the truth as probably was the conception of the world bv those who coined the catchy phrase.

There is a difference of about 4 per cent. which appears also in the apothecaries' ounce as against the fluid ounce, and is thus introduced in the smaller denominations, drams, grains, and minims; the fluid dram being equivalent to 56 grains, the minim about $96 / 100$ of a grain.

\section{INACCURATE DOSAGE.}

It is this very discrepancy or lack of commensurability which is so trying both to the physician and pharmacist. In the formulation of percentage solutions, where great accuracy is usually required, there will be a discrepancy of from 4 to 10 per cent., according to which particular method the quantities have been determined. If, for example, the formula appears:

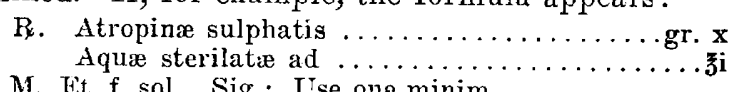

M. Et. f. sol. Sig.: Use one minim.

If prepared by fluid measure each minim will represent 1/48 grain of atropin sulphate; if, on the other hand, a troy ounce of water should be used, owing to the absence of the prefix "fl" before the ounce symbol, then the amount of atropin in one minim would be about $1 / 50$ grain, or, if perchance avoirdupois ounce be used, 1/44 grain would be contained in one minim. The difference in dosage may not be serious, but should such variations be tolerated, or such inaccurate methods be continued?

* Read at the Fifty-third Annual Meeting of the American Medical Association, in the Section on Materia Medica, Pharmacy and Therapeutics, and approved for publication by the Executive
Aside from the disadvantages of inaccuracy and lack of uniformity of the customary weights and measures, there is also the lack of correlation with our decimal system of notation. For domestic purposes the convenience of a decimal system of weights and measures, in harmony with our decimal system of coinage, must be equally as great as this so-called international system now appears to be advantageous for the extension of foreign trade. As is well known, the decimal system of weights and measures is now in use in all but Englishspeaking countries. As it is complementary to our decimal system of money, it should have been adopted along with it, as advocated by Jefferson and John Quincy Adams. The return to the old system of pounds, shillings, and pence would be no more absurd than to continue to inflict on the coming generation the present aggregation of weights and measures.

\section{THE FALLACY OF THE SPOONFUI.}

Contemporaneous with the customary weights and measures are the so-called domestic measures for liquids, as represented by the "spoonful." How this useful utensil varies in capacity is well known, and when it is also remembered that the amount of liquid a spoon will hold when "full" depends on the generosity of the person filling it, the spoonful method of administration, while innocuous for placebos, can not be regarded as scientific medication. The "meniscus," or the level of a liquid, which determines a measured quantity, is, according to the best authority (Dr. Hager), the medial of a transverse line drawn through the lowest point in the surface of the liquid and one drawn at the highest point of contact with the liquid and the confining sides. This line may be easily determined when the liquid is measured in glass, and the smaller the diameter the more exact the measurement. As is well-known, the graduated pipettes and burettes used in chemical work are constructed on this principle. In measuring by a spoon the meniscus can not be readily determined, and owing to the cohesion of liquids, especially when aqueous, after the spoon has been filled to its apparent level, an equal amount of the liquid can be poured on top of it, if done slowly, without any of the liquid running over. For these reasons it may be safely asscrted that instead of a teaspoonful being equivalent to one fluidram, it may be two fluidrams. In larger spoons the difference is proportionately less, but yet so great as to have no semblance of aceurate measurements. Not only should standard spoons be devised, but a uniform meniscus should, if possible, be adopted, although the spoon measure, as best being empiric, should be superseded by graduated glass measures, similar to those at present extensively used. A readjustment of the relative volumes of the three different spoon measures in vogue is necessary, and such relation should correspond with metric measures.

Recently the following metric equivalents have been approved by the Philadelphia College of Pharmacy, and incorporated in the scheme presented further on in this paper: One teaspoonful $=5$ c.c.; one dessertspoonful $=10$ c.c. $;$ one tablespoonful $=15$ c.c.

The following definition for the meniscus, originally taken from the French Codex, has also been approved: "A spoon is full when the liquid it contains comes up to, but does not show a curve above the upper edge or rim of the bowl."

THE DROP.
But if the spoonful measure is only an approximation, the drop shows still less uniformity in volume. As 
is well known, the size of drops varies with different liquids, is influenced by the size of the container and shape of the lip from which it is dropped, and by the temperature and volatility of the liquid. No careful physician would administer a potent agent bv drops except through a minim pipette. Recent investigations have shown that drops of uniform size may be obtained by dropping from a pipette of a certain outside diameter; thus a tube of $3 \mathrm{~mm}$. outer diameter will deliver 20 drops of water at $15 \mathrm{C}$., which will measure 1 c.c. when dropped at the rate of one drop per second. Nor is the size greatly influenced by the character of the liquid, be it watery or more mobile. Such standard "droppers" have been recommended for the exact measurement of medicinal liquids and, owing to its many advantages, this drop has been incorporated in the table.

\section{ADVANTAGES OF DECIMAL DIVISION.}

The decimal system has advantages in computing dosage and formulating combinations not possessed by the binary or octonary system on which our customary weights are based, because in accord with our system of numeration.
But it is in preparations for external use that the decimal system is sui generis, because their strengths are usually referred to by the per cent. or by per mille. Thus solutions for injections, etc., may be quickly prepared by taking for 100 c.c. the number of grams represented by the percentage strength; for 10 c.c. decigram may be used. Ointments, plasters, etc., may be equally readily computed.

\section{EXACT EQUIVALENTS THE OBSTACLE.}

The greatest obstacle to the more general acceptance of the metric system, especially for the older practitioners, has been the attempt to learn and to use exact equivalents for the customary weights and measures. Since the latter are not in accord with each other, the discrepancies appear in the respective metric equivalents, and the result is chaos and confusion. To illustrate: While 32 grams is a fair approximation to the apoth. ounce, it will not do to take it as equivalent to the fluid ounce, because in that event 16 fluid ounces would be equal to 512 gram, or one pint, considerable more than one-half liter, which at once makes the error apparent. This same discrepancy occurs in the other

ADAPTATION OF APOTHECARIES' WEIGHTS AND MFASURES TO THE METRIC SYSTEM.*

Showing by cross-references the relative quantities of customary denominations in Metric Terms, based on the Metric Standard Liter and Gram.

\begin{tabular}{|c|c|c|c|c|c|c|c|c|c|c|}
\hline 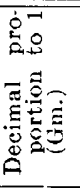 & Devomination. & 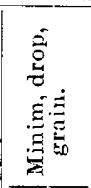 & 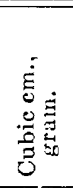 & 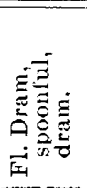 & 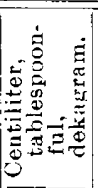 & 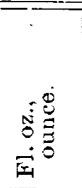 & 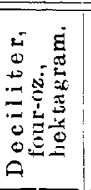 & 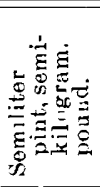 & 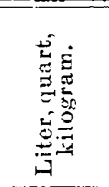 & Abbreviations in order of preference. \\
\hline 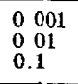 & $\begin{array}{l}\text { Milligram.... } \\
\text { Ceutigram... } \\
\text { Decigram. . }\end{array}$ & $\begin{array}{c}50 \\
5 \\
0.5\end{array}$ & $\begin{array}{r}1.000 \\
100 \\
10\end{array}$ & $\begin{array}{r}5,000 \\
500 \\
50\end{array}$ & $\begin{array}{r}10,000 \\
1,000 \\
100\end{array}$ & $\begin{array}{r}25,000 \\
2,500 \\
250\end{array}$ & $\begin{array}{r}100,000 \\
10,100 \\
1,000\end{array}$ & $\begin{array}{r}500,000 \\
50,000 \\
5.000\end{array}$ & $\begin{array}{r}1,000,000 \\
100,000 \\
10,000\end{array}$ & $\begin{array}{l}\text { mg.; } 0.001 \ldots . .1 \\
\text { cg.; ct g.; } 0.01 .\} \text { These three denominations given only } \\
\text { dcg.; dg.;0.1. }\end{array}$ \\
\hline $0.0 \overline{3}$ & $\begin{array}{l}\text { Minim, drop. } \\
\text { Grain........ }\end{array}$ & $\mathbf{1}$ & 20 & 100 & 200 & 500 & 2,000 & 10,000 & 20,000 & m.; mtm. ; min.; $\operatorname{mtt}_{.} ; 0.05$ c.c. \\
\hline 1 & Gubic cm.... & 20 & 1 & 5 & 10 & 25 & 100 & 500 & 1,000 & $\begin{array}{l}\text { c.c. } ; \mathrm{cm}^{3} ;: \mathrm{ccm} ; \mathrm{ml} . ; 1 \text { c.c. } \\
\text { Gm.; } \mathrm{G}, ; 1.0 .\end{array}$ \\
\hline j) & Fl. dram $\cdots$ & 100 & 5 & 1 & 2 & 5 & 20 & 100 & 200 & $\begin{array}{l}\text { fldr.; flmd.; flmtd.; met. spoonful; met. cochl. ; s c.c. } \\
\text { mdr.; mtd.; metdr, } 5.0 \text {. }\end{array}$ \\
\hline 10 & $\begin{array}{l}\text { Centiliter. } \\
\text { Dekagram } \\
\text { Fl ounce. }\end{array}$ & 200 & 10 & 2 & $\mathbf{1}$ & 2.5 & 10 & 50 & 100 & $\begin{array}{l}\text { cl,; ctlit.; met. tablespoonful; met. cochlmag.; } 10 \text { c.c. } \\
\text { IDkg.; Dekag.; } 10.0 \text {. }\end{array}$ \\
\hline 2j & $\begin{array}{l}\text { Ounce. } \ldots . \\
\text { Deciliter. . . }\end{array}$ & 500 & 25 & 5 & 2.5 & 1 & 4 & 20 & 40 & $\begin{array}{l}\text { mfloz.; met. floz.; quadcl.; } 25 \text { c.c. } \\
\text { moz.: imtoz.; quad. Hkg.; } 25.0 . \\
\text { del. decilit. }\end{array}$ \\
\hline 100 & $\begin{array}{l}\text { Hektogram... . } \\
\text { Semi-liter... }\end{array}$ & 2,000 & 100 & 20 & 10 & 4 & 1 & 5) & 10 & Hk, Hkg.; quatuncia; Four.ounce; 100.0 . \\
\hline 1060 & $\begin{array}{l}\text { Serni-kilogram. } \\
\text { Liter....... } \\
\text { Kilogram . . }\end{array}$ & 20,000 & 1,000 & 200 & 103 & 43 & 10 & 2 & $\mathbf{1}$ & 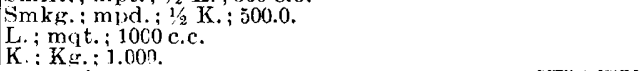 \\
\hline
\end{tabular}

This adaptation is based upon the fact that 20 drops of water at $15^{\circ} \mathrm{C}$, delivered from a minim pipette with an external diameter of $3 \mathrm{~mm}$., will measure one cc.

* From a scheme of adaptation presented to the Committee of Revision of the U. S. Pharmacopeia (1900) by the author.

A little practice will soon prove that by formulating the quantity in decimal amounts, the individual dosage may be seen at once without, as a rule, entailing any calculations. If, for example, ten or its multiple of powders, pills, etc., are ordered, the amount of the active agent for the whole quantity is at once apparent; thus:

R. Resinæ podophyllini..... 0

Hydrargyri chloridi mite. . 0 l

Sacchari lacti.......... $0 \mid 5$

R. Acidi arsenosi .......... $0[005$

Strychninæ .......... 0,001

Quininæ sulphatis ........ $0 \mid 1$

\begin{tabular}{|c|c|}
\hline \begin{tabular}{l|l}
0 & $\mathbf{l}$ \\
1 & 0 \\
5 & 0
\end{tabular} & \begin{tabular}{r|l}
0 & 2 \\
2 & 0 \\
10 & 0
\end{tabular} \\
\hline 10 pills & 30 pill \\
\hline & \\
\hline 001 & \\
\hline 10 & \\
\hline
\end{tabular}

If a liquid mixture is desired, it should be similarly ordered in quantities of 100 c.c., 50 c.c. or 25 c.c.

Dose in Dose in Dose in

5 c.e. $\quad 100$ c.c. 50 c.c.

R. Morphinæ sulphatis...... 0001

Potassii bromidi ........ 00

Chloralis ............. 033

Syrupi aurantii ad........ $\left.5\right|_{0} ^{3}$

\begin{tabular}{r|ll|l}
0 & 2 & 0 & 1 \\
10 & 0 & 5 & 0 \\
6 & 0 & 3 & 0 \\
100 & 0 & 50 & 0
\end{tabular}

denominations: dram, grain, and minim, and is too serious an objection to be disregarded.

There is only one way of using the metric system, and that is to use it, or a decimal adaptation of it, combining the desirable units, grain and minim, which are so inseparably bound up with dosage in the medical mind.

With 20 drops, equivalent to a cubic centimeter, instead of 16 drops, and assuming the gram to contain 20 grains instead of 15.432 grains, we obtain at once these two essential denominational units as concordant and with the necessary decimal relation to the metric units, cubic centimeter and gram, on which a simple adaptation of the customary weights and measures may easily be constructed, as shown by the accompanying table.

As will be observed, there are no changes whatever in the metric standards.

The new metric grain proposed is about one-fifth less than the troy grain.

The minim is a little less than one-fifth smaller. 
The new dram is about two-ninths greater than the apothecaries'.

The new fluidram is about three-elevenths larger than the present fluidram.

Both drams contain 100 grains or minims.

The new ounces would be all alike, representing 25 gm. or c.c. and would be from one-eighth to one-fifth less than the customary ounces.

The metric ounce, 25.0 ; one-fifth less than 1 apoth. ounce, 31.0 ; one-sixth less than 1 av. ounce, 28.35 .

The metric fl. ounce, 25 c.c.; two-thirteenths less than 1 fl. ounce, 29.5.

The metric pint and pound are 5 to 10 per cent. greater respectively than the pint and avoirdupois pound.

The discrepancy in these two is not great, and would be readily adjusted. Each would contain 20 ounces; or 100 drams, or 10,000 grains, or minims.

The advantages of this adaptation for the calculation of doses, the strength of preparations, etc., is self-evident. The dose of an active agent in any quantity of a solution or mixture would have a definite decimal proportion and could be quickly calculated in metric grains, or minims. The smaller quantities represented by the proposed grain and minim would not be as objectionable as is the lack of proper relation between the present denominations - a fruitful source of error in practice. Besides, the difference is on the safe side, and by employing the proper designations no mistakes could occur. The author recommends that the abbreviation of "Gm." for gram should always be written with a capital G, to avoid confusion with "gr." for grain, which is never written with a capital letter. The metric fluidram is especially designated "metric spoonful" to afford an accurate method for administration of liquid medicines.

The adoption of a definite spoonful measure would cause the domestic measurement of medicines by graduated measures exclusively. Of the centiliter, or tablespoonful, the same may be said. The decimal proportion of the ounces to the drams and also to the grain and minim would enable the prescriber to at once estimate the quantities and yet have the metric equivalents at a glance. The deciliter, or four-ounce, would be an exceedingly convenient quantity for extemporaneous prescription; containing $20 \mathrm{fl}$. drams it is well adapted to formulas of which the dose is a teaspoonful. For example: one grain at a dose; then 20 grains $=1 \mathrm{Gm}$. to 100 c.c., or 4 fl. ozs.

In the belief that the adoption of some such adaptation, possibly as an appendix in the U. S. Pharmacopeia, would materially aid in the more general acceptance of the metric system for prescription writing, this scheme has been proposed to the Committee of Revision and is now presented to the profession.

NoTw.-This paper was referred to a committee consisting of Drs. Frank Woodbury, H. C. Wood, Jr., and A. A. Eshner of Philadelphia to report on at the next annual session.

A remarkable specimen was shown at the meeting of the "Section on Clinical Medicine and Surgery" of the Medical and Chirurgical tiaculty, December 19 , by Dr. José Hirsh. It was an immense aneurism of the arch of the aorta of 24 years' standing. The sac was entirely filled with a hard clot and had projected forward, evading the sternum at the junction of manubrium and gladiolus, so that these bones were at right angles to each other. Notwithstanding his condition, the man (a Frenchman) had led a laborious life as a bricklayer. The case was considered one of spontaneous cure. Owing to the position of the tumor, there was a remarkable lack of symptoms. Oozing of blood from the apex of the tumor took place from time to time, and infection occurred in this way, death being due to general streptococcus infection.

\section{THE IDENTITY OF NERVE FORCE AND ELECTRICITY.* \\ J. EMMET O'BRIEN, M.D. \\ SCRANTON, PA.}

There has been in recent years such advance by physiologists in knowledge of nerve mechanisms, though not of nerve force, and such advance by electricians in knowledge of electricity in its protean phases, that, I think, the view of the relation of nerve force to electricity ought to be taken up anew by physiologists and restudied in the light of modern electricity. Advances by physiologists appear in minutely studied anatomy of neurons, relaying nerve mechanisms, conduction paths, terminal mechanisms, etc., and advances in this direction by electricians have been in better knowledge of the electric phenomena of cables, learned since physiologic views of the electrical phenomena of nerves became rather arbitrarily fixed; of fine currents and the wide field of induction, as revealed by the telephone; new light on the merely relative value of insulation; the phenomena of high frequency currents and their apparent reversal of electric law in insulation; storage or secondary batteries; electric relays, repeaters, transformers, microphones, terminal mechanisms, etc., and lastly electrolysis and electrochemistry.

\section{PRIORITY AS TO THIS THEORY.}

Noting the analogies between nervous and electric mechanisms revealed by the advances of recent years in the separate fields of physiologic anatomy and electricity, I affirmed the identity of nerve force and electricity in a paper" entitled "Analogies Between Nervous and Electrical Mechanisms: What is Nerve Force?" to our county and state medical societies in 1900 . On account of the importance of the subject, and because the same conclusion has since been reached through other paths by Professors Loeb and Mathews, I again present it, with some further studies. The lay press has, as you are no doubt aware, given entire credit for originality, not only in methods, but as to scope and conclusions, to Professors Loeb and Mathews. They are entitled to great credit for methods in arriving at the identity of nerve force and electricity. ${ }^{2}$ But it is not the lay press but scientific societies and journals that ought to adjudicate the question of originality in method, scope and conclusions; and so to you I leave it.

\section{THE SPEED OF NERVE IMPULSE TRANSMISSION.}

In reviewing works on physiology $I$ have not found reasons given for the usual statement that nerve force is not electricity. I presume that the supposed slow speed of nerve force, the apparent want of insulation, of continuous circuits and of galvanic elements in nerves have been the principal objections. If there are other, better reasons I am anxious to know and acknowledge their validity. It does not appear that any very decisive experiments indicating slow speed of nerve impulse have been made since those of Du Bois Reymond and Helmholtz, though theirs have been repeated by many. Although we have since learned much about electricity, these experiments appear to bind opinion still. These experiments were made and interpreted before much was known of the electric phenomena of cables; before

* Read at the Fifty-third Annual Meeting of the American Medical Association, In the Section on Pathology and Physlology, and approved for publication by the Executive Committee: Drs. A tengel, Winfeld $\mathrm{S}$. Hall and Frank 1 . Wynn.

1. Published with drawings in the Pennsylvania Medical Jour, November, 1900 . 2. See, for instance, "The Nature of the Nerve Impulse," by
Mathews, Century Magazine, March, 1002. 